\title{
CATS AND HUMAN DIPHTHERIA.
}

\author{
By WILI.IAM G. SAVAGE, M.D., \\ County Medical Officer of Health, Somerset.
}

IT is a widely accepted belief amongst medical men, particularly those who are Medical Officers of Health, that cats may suffer from diphtheria and convey it to human cases and that they are a not uncommon source of infection. It is of considerable practical importance to determine to what extent this belief is based upon reliable scientific data or whether it is another of those opinions, still far too prevalent, which arise from the acceptance of insufficiently tested and incorrectly interpreted observations.

In dealing with this subject I propose to give first a brief account of the available evidence upon which the association of cats with diphtheria has been built, then a summary of my own investigations and lastly a critical consideration of the whole of the data available.

\section{EVIDENCE ASSOCIATING CATS WITH HUMAN DIPHTHERIA.}

This is both experimental and epidemiological.

Of the experimental work the most considerable in volume and of most importance as regards its influence upon contemporary and later medical opinion is the work of Klein $(1888,1889,1890)$. This investigator inoculated eight cats subcutaneously and two intravenously with pure cultures of diphtheria bacilli. Four were unaffected but the other six showed swelling at the site of inoculation followed by death after five to eleven days. Postmortem all the fatal cases showed extensive haemorrhagic oedema locally and certain special kidney changes characterised by enlargement of the whole organ, almost the entire cortex being grey and fattily degenerated, the medulla appearing by contrast much congested. Microscopically the uriniferous tubules were swollen and the epithelium showed extensive fatty degeneration or broken down into a granular debris. Klein describes this condition of the kidney as extremely characteristic of diphtheria disease in the cat. The condition of the supra-renal capsules is not mentioned.

He also caused death or paralysis in two cats by injecting diphtheria bacilli direct into the trachea by a syringe pushed through the anterior wall.

One kitten and two cats fed with agar cultures in milk remained well, but two other cats fed on three separate occasions became very thin, although showing no other symptoms, and were killed three and four weeks after the start of the experiment. Both showed livers much enlarged, in one the kidneys 
were large with white cortex, in the other the kidneys were not conspicuously large but their cortex was pale with a few whitish patches. Not the slightest bacteriological proof is given or evidence supplied of the presence of any clinical or pathological features of diphtheria but Klein remarks "From these experiments it is seen that by repeated feeding with cultures of diphtheria bacilli distributed in milk, unquestionable diphtheria disease can be produced in the cat." Klein also laid stress upon certain cases of accidentally acquired diphtheria (so called) in cats. The symptoms were an acute catarrhal affection of the conjunctiva and of the respiratory passages. The diagnosis of diphtheria was based upon the pathological appearances of the kidney and the appearance in one of the cats of a grey membrane covering the lower part of the larynx and upper part of the trachea in which "the microscope showed crowds of diphtheria bacilli as smaller or larger groups in the necrotic mucous membranes." Diphtheria bacilli were also demonstrated in the bronchial and tracheal exudation of another cat. Nine months later a somewhat similar outbreak occurred with similar pathological lesions and the presence of bacilli morphologically like diphtheria bacilli.

Klein records his examination of three cats sent to him from different sources and all associated with human cases of diphtheria. All three cats had been ill. The kidney condition which Klein records as typical of diphtheria was found in each case but no diphtheria bacilli were cultivated. On the basis of the pathological appearances and their likeness to the conditions induced in cats by inoculation with Klebs-Löffler bacilli Klein considered these were cases of cat diphtheria.

Renshaw (1885) in 1874 administered diphtheric membrane from human cases to a number of cats. Most died after illness and Renshaw reported finding diphtheritic membrane lining the fauces, bronchial tubes, etc. No evidence was adduced that the lesions were due to diphtheria bacilli and not associated with pathogenic cocci, etc.

Welch and Abbott (1891) inoculated into the trachea of a half-grown kitten a platinum loopful of a pure culture of the diphtheria bacillus. The animal died on the third day with the production of a diphtheritic membrane, containing diphtheria bacilli, in the trachea and larynx. No other noticeable lesion was found "unless it be a greater degree of fatty metamorphosis of the renal epithelium than is normal in kittens."

The above constitutes the only experimental work I have been able to trace.

Evidence is also available on the epidemiological side in the form of instances in which it is reported that the disease has been transmitted to human cases through the agency of cats. Without making a thorough search of the literature I have collected particulars of a number of such cases.

Turner (1886) appears to have been the first to report the association of illness amongst cats with cases of human diphtheria and he drew attention to their presence in a number of small outbreaks. The evidence was entirely 
circumstantial and consisted of the concurrent presence of human cases with cats ill with such symptoms as swelling of the neck, foul discharge from the nostrils, eye inflammation, etc.

Bruce Low (1888) reported a similar relationship in an outbreak at Enfield.

Williams (1895) reported that in the same house as a case of human diphtheria three cats were taken ill with wasting, loss of appetite, inability to swallow, cough and expectoration and all three died. One cat was sent to Klein for examination who reported that there was extensive disease of the lung not uncommon amongst cats.

Gwynn (1893) reported at Hampstead the association of a case of human diphtheria with an ailing cat suffering from a bad swollen throat. Later it showed an unhealed abscess in the vicinity of the throat. No bacteriological examinations were made.

Dowson (1895) examined a cat which became ill in a house in which a child had died 14 days earlier from diphtheria. Cultures from the lung gave a pure culture of an organism morphologically resembling the diphtheria bacillus which was not further investigated.

Symes (1896) mentions a kitten which was in close and constant contact with a fatal case of diphtheria and which subsequently was attacked with vomiting and diarrhoea with slight swelling of the neck and with patches of yellow exudation on the fauces. These patches extended, the cat became nuch emaciated and died. Unfortunately the cat was buried before bacteriological examinations could be made.

Barras (1905) reports that in two cases of diphtheria in Govan a history of illness affecting the cats of the families attacked was ascertained. The throats of two of these cats were examined in the laboratory and nicro-organisms were cultivated on artificial media which were found to correspond in every respect to those of the human diphtheria bacillus. No particulars are given of the tests employed for the identification nor are any details furnished as to the degree or nature of the illness from which the cats suffered. Barras also mentions another cat in Govan-a stray cat-which had obtained a home in one of the families affected and which was found to be suffering from postdiphtheritic paralysis of the legs. Apparently no bacteriological examinations were made.

Porter (1908) records a number of cases of diphtheria in a household containing two petted and favourite cats. The cats were not ill. Swabs were rubbed over the fur of each cat. One yielded negative results but smears from the culture from the other cat showed a considerable number of organisms identical in appearance with the Klebs-Löffler bacillus both when stained by methylene blue and by Neisser's method. Porter was unable to isolate the organism in pure culture.

Mapleton (1913), in connection with an outbreak of diphtheria amongst the children living in the Cottage Home, Newton Abbot, reported that there were "three cats in this Home in somewhat intimate association with the chil- 
dren and a bacteriological examination of these proved that two of them were infected." Further inquiries showed that the examinations made were ordinary swabbings grown on blood serum and that the bacilli were not isolated in pure culture nor were any animial inoculations made. In this case the cats were not definitely ill although one was obviously not in health and were not suspected of having conveyed any infection. They were examined to prevent the possibility of their carrying infection elsewhere.

Webb (1914) reported an interesting outbreak at Leigh (Lancashire) amongst cats to which his attention was directed in connection with a case of diphtheria in a child. In the house with this human case the cat had been ailing for more than two weeks, had a peculiar cough, could not miaw properly, refused food, was very thin and could hardly move. During the previous week a cat next door had died presenting the same symptoms and a cat across the way had also died with the same symptoms.

The throat of the cat in the house with the diphtheria case was in a dirty sloughy condition and cultivations showed "a bacillus having the characteristics of the Klebs-Löffler bacillus." The organism was not isolated in pure culture and no cultural or inoculation tests were carried out. Webb states that five other cats in the street were found to be ailing and were destroyed.

Priestley (1915) investigating a number of cases of diphtheria at the Stockwell Orphanage found nine cats in that institution. All were bacteriologically examined although none of them were apparently ill. He reports "three of them in the boys' department and one belonging to the girls' department carried the diphtheria bacillus." In a personal communication he was kind enough to inform me that the bacilli were morphologically and culturally undistinguishable from the true Klebs-Löffler bacillus. No inoculation experiments were carried out nor is any information available as to what cultural tests were employed but apparently they were not isolated in pure culture and then tested.

The significance of the above data will be considered after my own experiments have been recorded.

\section{THE AUTHOR'S INVESTIGATIONS.}

These have been along three lines of inquiry:

A. The bacteriological examination of the throat and nose of healthy cats not associated with any cases of human diphtheria.

B. The bacteriological and pathological examination of cats associated with human cases of diphtheria.

C. Experimental investigations with kittens.

\section{A. Examination of Healthy Cats.}

Eight cats and 12 kittens were examined, the throat being examined in every case while for most of the kittens and for a few of the cats the nose was also swabbed. One cat was examined twice. The method of examining 
consisted in taking one or more swabs in the ordinary way and then charging at least three blood serum tubes and one agar tube in series without recharging. The usual order was 1 serum, 1 agar, 1 serum, 1 serum tube, the object being to obtain considerable dilution of bacteria on the last two serum tubes so that discrete colonies could be obtained. In some cases pea-flour trypt-agar plates, a medium upon which diphtheria bacilli grow well, were also inoculated. All the 12 kittens failed to show any bacilli which at all resembled diphtheria bacilli.

The eight cats were very different. Swabs from three of them showed no bacilli which might be taken for Klebs-Löffler bacilli. The results from the other five were as follows. The characters refer to films stained by methylene blue.

(1) Short bacilli, well marked beading chiefly at ends but some with central staining. Some curved and longer forms very closely resembling KlebsLöffler bacilli. Bacillus isolated in pure culture.

(2) A number of bacilli present closely resembling Klebs-Löffler bacilli, thin, curved some thicker at ends and a few, but not the majority, showing polar staining. Do not show granules when treated with acetic acid. Isolated in pure culture.

(3) A large number of bacilli present resembling Klebs-Löffler bacilli. Curved, beaded but not definitely clubbed. No dark blue granules when treated with acetic acid. A bacillus resembling this organism isolated in pure culture.

(4) Numerous bacilli present which are curved, thin and beaded. Not thickened at ends but closely simulate Klebs-Löffler bacilli. Isolated in pure culture.

(5) A very mixed growth but contains a number of bacilli which very closely resemble true diphtheria bacilli being long, curved bacilli with granules at end but not in the middle. Granules show up well when the films were treated with acetic acid. Differ from typical Klebs-Löffler bacilli in being rather more uniform in size and the absence of clubbing. Was unable to isolate in pure culture.

No. 2 is of particular interest as it was the cat in a large girls' school. This cat was re-examined exactly three months later. $=2 a$.

(2 a) Many bacilli closely resembling Klebs-Löffler bacilli present, being curved, and granular, thicker at one end and showing dark blue granules when the film was treated with acetic acid. Isolated in pure culture.

The bacilli isolated from cats (1), (2) and (3) were all alike so far as they were culturally investigated. They morphologically resembled true diphtheria bacilli less when isolated in pure culture although still superficially resembling it. Culturally they were distinct, the agar and blood serum growths being definitely yellow or yellow-white in colour while they produced no acid in glucose media and in litmus milk produced definite alkali after four to five days' growth.

The bacillus from cat $(2 a)$ was extremely like the true diphtheria bacillus 
both morphologically and culturally. It produced acid in glucose broth and grew like the diphtheria bacillus in milk. It was only distinguished so far as it was tested by the blood serum colonies having a distinct yellow tinge. Morphologically it was indistinguishable. It was non-pathogenic to a guinea pig.

It may be added that the films from the serum cultures made direct from the swabs were shown to the county bacteriologist in the laboratory who was examining several thousand suspected diphtheria swabs every year and he as well as myself was unable to distinguish the films from those from swabs from human throats containing true diphtheria bacilli.

We have therefore the striking fact that two experienced bacteriologists were unable to distinguish with any certainty these bacilli in mixed smears from true diphtheria bacilli although in several instances we were in some doubt and could not pronounce them typical bacilli since minute differences were apparent. In several cases the resemblance was extremely close. All of them however (with the possible exception of the strain from cat $(2 a)$ ) were definitely not true diphtheria bacilli. Present in 66 per cent. of the adult cats it is interesting to note their entire absence from all 12 kittens.

\section{B. Examination of Cats associated with Human Cases.}

Although through Public Health medical officers of health were specially asked to send me such cases, none reached me in this way and the few cases investigated were all obtained through my personal efforts. Four were investigated in 1916 and one in 1919.

Case 1. Case of severe diphtheria in a house. A favourite cat much in the room with this case and with two children who suffered from severe colds but whose throats showed no diphtheria bacilli when swabbed.

The cat showed no signs of sore throat or other illness. Swabs from its throat were implanted on several blood serum tubes in series. The films showed numerous bacilli which in the primary films could not be distinguished from true diphtheria bacilli either by myself or the laboratory bacteriologist. A short form with marked polar staining. Isolated in pure culture the bacillus decidedly less closely resembled the Klebs-Löffler bacillus morphologically, while the yellow colonies and absence of acid production in glucose clearly showed it was not that organism.

If morphological appearances from the mixed growth swab had been relied upon undoubtedly this cat would have been classed as infected with diphtheria bacilli and would probably have been credited as the source of the infection.

Case 2. A human diphtheria case (a woman of 21) in a house. The house cat came into close association with this woman who was very fond of the animal. The cat was said to have been ailing for some days before the case was notified but no very clear particulars as to symptoms were forthcoming. 
The cat when examined showed no local or general symptoms and the bacteriological examination of the throat swab showed a few bacteria suspiciously like diphtheria bacilli but nothing more definite.

Case 3. The cat in the house was closely associated with a case of human diphtheria. The animal remained throughout quite well and the cultures from the swab from its throat showed no bacilli at all like true Klebs-Löffler bacilli.

Case 4. A boy of eight years developed diphtheria on Nov. 22nd, 1916. The house cat was said to be "off its food" for about a week before that date and subsequently showed difficulty of swallowing as if it had a sore throat. The patient was very fond of this cat and fondled and nursed her regularly.

The cat was swabbed Dec. 6th. The films made from the blood serum cultures showed a few clusters of bacilli which morphologically rather resembled diphtheria bacilli of the short type. They were slightly curved, beaded and thicker at one end but rather thick compared with the true organism. Impossible to exclude as not Klebs-Löffler bacilli. They were only present in very small numbers and could not be isolated in pure culture. I was fortunate in being able to secure the cat and re-swabbed it in the laboratory but the cultures from this swab failed to show any bacilli suspicious of Klebs-Löffler bacilli.

The cat was killed and a postmorten examination made. There were no pathological lesions, the kidneys and other organs being quite healthy. Further swabs from the back of the throat and from the upper part of the trachea showed culturally no bacilli resembling diphtheria bacilli.

It may be mentioned that the cat when the first swab was taken and when later on examined at the laboratory showed no symptoms at all of ill health.

Case 5. A child, aged five, was removed to the isolation hospital suffering from diphtheria the diagnosis being bacteriologically confirmed. The child was said to have been ailing for at least ten days before removal. An elder sister (16 years) also had a sore throat before the notified case and was possibly the source of infection, but when swabbed the day the notified case was removed to hospital showed no diphtheria bacilli. According to the mother of the case the house cat was ill for 3-4 weeks before the child of five was notified, refusing to eat and with some discharge from the nostrils and mouth. The cat however gave birth to some kittens soon afterwards and then recovered and was quite well at the time of the removal of the case to hospital. The cat was brought to the laboratory April 16th, i.e. six days after the removal to hospital of the case, and swabbed. It showed no signs of any illness or nasal discharge. The animal was killed and postmortem showed no membrane in the throat or any lesions anywhere.

The films from the blood serum cultures made showed (mixed with abundant cocci) bacilli which were beaded, curved and thicker at one end and which could not be distinguished from diphtheria bacilli. With great difficulty this organism was isolated in pure culture and investigated. Morphologically 
it could not be distinguished from the diphtheria bacillus and the blue granules after treatment with acetic acid were very marked. Culturally it grew like diphtheria bacilli on ordinary agar and blood serum but unlike our ordinary diphtheria strains more abundantly on the former than on the latter. Indeed it did not grow well on blood serum. It produced acid in glucose broth but none in lactose or mannite broth while it produced a trace of acid in saccharose broth.

To test its pathogenicity a guinea pig was inoculated subcutaneously from a broth culture reinforced by the growth on a tube of pea-flour trypt-agar (i.e. a very heavy dose) but the animal was unaffected.

The cultural characters agree with the diphtheria bacillus for the most part and this may possibly have been a non-pathogenic Klebs-Löffler bacillus.

\section{Experimental Investigations with Kittens.}

If, as has been so often asserted, cats suffer from diphtheria or even if it be merely advanced that they act as carriers of the diphtheria bacillus in their throat or nose, it should be possible to infect them artificially and set up either condition.

The possibility of this was tested in a long series of experiments. Kittens were used in preference to adult cats as both more easily handled and as likely to be more susceptible to infection than the grown up animals. In every case the throat and in nearly all cases the nose of the animals were swabbed before the experiments started to ascertain if diphtheria-like bacilli were present or absent.

The kittens were all kept in separate cages and very carefully examined for any signs of ill health. Weight observations were recorded but temperature testings were not found very helpful. The kittens were usually from four to six weeks old when the experiments started.

Exp. 1. Throat swabbed with, as far as possible, all the growth of a blood serum culture of $B$. diphtheriae transferred on a sterile swab. Immediately re-swabbed in the same way with a second blood serum culture of another strain.

Throat swabbed after three and seven days and several blood serum tubes inoculated. No diphtheria bacilli found. Animal showed no signs of any illness, quite lively and gained in weight. No local throat-lesions.

Exp. 2. Throat of same kitten re-swabbed a month after onset of Exp. 1 with as far as possible the whole of a blood serum culture made direct from a swab taken from the throat of an acutely ill case of diphtheria. This culture showed about half the colonies $B$. diphtheriae and half streptococci and it was used as possibly the mixed growth might favour infection. Animal remained quite well and lively and a swab taken six days after the inoculation showed no diphtheria colonies on the several blood serum tubes inoculated.

Animal killed eight days after the second inoculation. Postmortem all the organs were healthy and sections of kidney and supra-renals showed no abnormalities. No trace of any lesions in the throat.

The virulence to guinea pigs of these three strains was not tested. 
Exp. 3. Kitten about four weeks old fed on two consecutive days with milk containing the whole of two blood serum growths of two different strains of $B$. diphtheriae, one of which was fully virulent to a guinea pig. Animal showed no symptoms whatever and the throat swabbed six days after the first feeding yielded no diphtheria bacilli.

Exp. 4. Throat of the same kitten swabbed with, as far as possible, the whole of two blood serum cultures of $B$. diphtheriae (plus a small number of staphylococcus colonies) grown direct from the throat of an acute case of diphtheria. Throat swabbed seven days later showed no diphtheria bacilli. Animal showed steady increase in weight and no local or general symptoms.

Kitten killed 22 days after onset of the feeding and 15 days after the throat swabbing. No trace of membrane on throat, trachea or larynx. Internal organs normal and sterile. Sections of the kidney and supra-renals showed no pathological changes except some engorgement of the kidney blood vessels.

Exp. 5. Throat of a kitten about five weeks old swabbed with the whole of a blood serum growth from a fifth strain (strain B). This blood serum growth was direct from a swab from an acute case and showed $B$. diphtheriae in almost pure culture. Swabs from the throat taken 4 and 10 days after inoculation showed no diphtheria bacilli. The animal increased in weight and exhibited no signs of ill health.

Exp. 6. Thirteen days after Exp. 5 started this kitten was injected subcutaneously with 2 c.c. of a five days old (trypsin pea-flour) broth pure culture of the same strain (strain B) of diphtheria bacillus. The animal was obviously ill within 24 hours of the injection and remained very quiet. The temperature which for the two days before the experiment had fluctuated between $99^{\circ}$ and $100^{\circ}$ rose to $104^{\circ} \mathrm{F}$. the morning and also the evening after the inoculation. It remained between $102^{\circ}$ and $104^{\circ}$ for seven days, then rapidly dropped to below $100^{\circ} \mathrm{C}$. and was $96^{\circ}$ on the ninth day when the animal was moribund and was killed. The day before death very definite paralysis of the hind legs was observable. The postmortem examination showed no oedema or inflammation at the site of inoculation, no fluid excess in the thoracic cavity. The internal organs, apart from the kidneys and supra-renals, appeared healthy. Both kidneys were greatly enlarged and in section showed marked cloudy swelling of the cells of the cortex and medulla with enlargement of all the blood vessels. The supra-renals showed a condition of acute inflammation with dilated engorged blood vessels and swollen, turbid and indistinct cells in both cortex and medulla.

This experiment is important as showing that the same strain which was incapable of infecting or affecting the kitten by means of the throat was highly pathogenic when injected into the same animal.

Exp. 7. A rather older kitten (about two months) and weighing 830 grms. used for a combined throat swabbing and feeding experiment.

Throat swabbed with the whole of a blood serum culture of another 
B. diphtherice strain (strain J). Throat examined after two, five, and nine days showed no diphtheria bacilli.

Five days after the third inoculation fed with milk in which strain $J$ had been grown for 24 hours fortified by the whole of a 24 hours growth on blood serum of the same organism.

Feeding with the same massive dose repeated on the following three days. Throughout the animal remained quite well and gained steadily in weight.

Exp. 8. The same kitten some weeks later injected subcutaneously with 1.8 c.c. of a two days glucose broth culture of strain J. The kitten showed a slight rise of temperature but no other symptoms and increased in weight. Killed 14 days after the injection showed no macroscopic or microscopic lesions. The growth in the broth culture was scanty and the kitten weighed when inoculated 1020 grms. so the dose was probably inadequate and this experiment hardly indicates the strain was non-virulent.

Exp. 9. The nose of a young kitten about four weeks old inoculated heavily with a pure culture on blood serum of $B$. diphtheriae (strain W) transferred on a thick blunt platinum needle. Previous swabbings of nose (both sides) and throat showed absence of any diphtheria bacilli-like organisms.

Nose swab examined 24 hours after the inoculation failed to show any diphtheria bacilli when grown on blood serum tubes. Both sides of the nose then again inoculated with $B$. diphtheriae in the same way as before and each nostril examined after 48 hours and eight days showed no diphtheria bacilli.

The animal remained perfectly well with no nasal discharge or any symptom.

Exp. 10. It may be argued that the failure to infect the kittens by the throat was due to the smooth healthy mucous membrane failing to afford a nidus for growth and the following and some other experiments were devised to deal with this objection.

Thirteen days after the onset of Exp. 9 the throat of this kitten was well painted with a 20 per cent. solution of silver nitrate. Examined 24 hours later there was some reddening of the throat and superficial ulceration of the roof of the palate. Throat re-painted with the silver nitrate solution. After a further 24 hours there was definite redness with much mucous and some whitened areas on the palate. The throat was then inoculated with, as far as possible, the whole of a two days old blood serum culture of $B$. diphtheriae (strain W). Swabs from the throat 24 hours and 48 hours after the $B$. diphtheriae implantation when grown on blood serum showed no diphtheria organisms. The animal remained fairly well but was quiet and did not put on much weight but gradually recovered completely.

Exp. 11. Eight days after the start of Exp. 10 this kitten was injected subcutaneously with 2 c.c. of a 24 hours broth culture (pea-flour trypt broth) of the same B. diphtheriae strain (strain W). Animal obviously ill 24 hours later. After 48 hours very ill, quiet and refused food. Three days about the same and after four days appeared better but died on fifth day. 
Postmortem the animal showed the signs noted above as present in the other injected kitten except that the reddening of the supra-renals was trifling or absent. They showed however inflammation signs when sections were made and stained. The kidneys were especially engorged and enlarged. Exps. 10 and 11 show that this strain while highly pathogenic when injected was unable to establish itself upon the throat of the same animal even when a definite unhealthy and pathological nidus was provided.

Exp. 12. The throat of a young kitten swabbed with as far as possible the whole of a two days old blood serum growth of $B$. diphtheriae (the virulent strain $\mathrm{W}$ being used). Nose (left side) also inoculated from another serum culture of the same organism. No diphtheria bacilli found in the throat when examined after 24 and after 48 hours. Diphtheria bacilli found in the nose at the end of 24 hours but not after 48 hours or subsequently. Animal remained quite well.

Exp. 13. Nose and throat of another quite young kitten inoculated heavily from another $B$. diphtheriae strain (strain V). Both nose and throat were free from diphtheria bacilli when examined after 24 and 48 hours.

Exp. 14. This same kitten was then fed on eleven occasions over 14 days with a pea-flour broth culture of $B$. diphtheriae (strain W). This broth gave abundant growth and over this period as much as 100 c.c. of broth culture was administered. No illness or symptoms of any kind. The animal steadily increased in weight and the postmortem examination $3 \frac{1}{2}$ weeks after the commencement of the feeding showed no abnormalities.

Exp. 15. The hard palate (as far back as possible) of another young kitten was scarified by a scalpel making a number of superficial lesions. A B. diphtheriae (strain W) blood serum culture transferred on a sterile swab was then at once well rubbed into these superficial lesions. The animal remained quite well and examined 24 and 48 hours after showed no membrane or other lesions nor could diphtheria bacilli be cultivated from the swabs taken although a good many tubes were used.

Exp. 16. Throat of a young kitten swabbed with a strong solution of silver nitrate. Examined 24 hours after there was a well marked slough. The throat was inoculated with a blood serum culture of diphtheria bacilli. The animal was very ill and as it was in pain was killed. Here the throat treatment was too severe but swabs after 24 hours showed no diphtheria bacilli and postmortem there was no evidence of any true membrane although sloughs over the hard palate. Films from throat and trachea showed no bacilli like B. diphtheriae.

Exp. 17. The nose of another young kitten inoculated heavily with B. diphtheriae (strain W). Nose swabs taken previously showed no bacilli at all like diphtheria organisms. Nose examined after 24 hours and after 48 hours showed diphtheria bacilli colonies on the blood serum tubes and apparently more abundant after the longer period. None found after four and five days. Unfortunately not examined after three days. The animal 
remained perfectly well, gained in weight, had no nasal discharge and postmortem showed no lesions.

Exp. 18. Nose of another kitten inoculated heavily with two separate strains of $B$. diphtheriae (strains $\mathrm{W}$ and $\mathrm{V}$ ).

After 24 hours diphtheria bacilli readily grown from the nose. Found but less readily after 48 hours. Examined after four days the bacilli were found in the cultures but with great difficulty and could not be found at all after five and six days. The kitten remained perfectly well throughout, increased in weight and showed no nose discharge or other local lesions.

Exp. 19. The nose of another kitten inoculated heavily with another $B$. diphtheriae strain (strain $\mathrm{R}$ ) isolated a day or two previously from an acute case of diphtheria. After 24 hours a few diphtheria bacilli were grown from the nose but none after 48 hours, four and five days. Animal remained perfectly well without any local or general symptoms.

\section{Remarks on the Experiments.}

The results obtained with these young kittens are exceptionally uniform and concordant. It was found impossible to infect them by throat swabbing although very massive doses were invariably used, as many as 10 different strains employed and all of them were quite recently isolated from acute human cases of diphtheria. Further, four of the strains used were proved to be of high virulence and two definitely killed, when subcutaneously injected, young kittens they were unable to infect by natural channels of entry. The same failure to infect these animals with diphtheria or any local lesion was experienced when mixed cultures direct from human throats were used or when an artificial nidus for local growth was provided by chemical or mechanical means. Not only did the bacilli not infect but they failed to survive even and although such massive doses were inoculated they invariably disappeared after as short a period as 24 hours.

The implantations of vast numbers of diphtheria bacilli into the nasal cavities were equally ineffective in setting up any local or general lesions and (what I did not expect to find) were unable even to survive beyond a very short period. In only one experiment was there possible evidence of increase and only in one instance could the nost diligent examination trace their persistence beyond four days.

The feeding experiments were also unsuccessful and the kittens consumed vast numbers of virulent diphtheria bacilli without the slightest evidence of harm.

These experiments so far from supporting the view that diphtheria is a naturally occurring disease of cats suggests that the secretion from the mucous membranes of the cat are peculiarly unfavourable to the growth of the diphtheria bacillus and will not even permit it to remain as a saprophyte. 


\section{CRITICAL CONSIDERATION OF THE AVAILABLE FACTS.}

There are two separate possibilities. On the one hand there is the suggestion that cats themselves may be affected with disease due to their infection with the diphtheria bacillus, and which therefore may be appropriately designated as cat diphtheria, while on the other hand we have to consider the possibility that these animals may act as carriers of diphtheria infection by harbouring the bacilli in their nose or throat or by their fur becoming infected.

As regards the first possibility it must be accepted from the work of Klein and others, corroborated by my own experiments, that cats are not immune to the toxins of $B$. diphtheriae and that when these are introduced under the skin or by other means such as direct injection into the trachea fatal results may occur with the development of pathological lesions not dissimilar from those which are observed when guinea pigs or other animals are similarly injected. This of course no more proves or even suggests that cats suffer from diphtheria than it does that guinea pigs so suffer or the latter animals from typhoid fever because they succumb to the injection of typhoid bacillus toxins.

A study of the experimental data in favour of cats suffering from diphtheria detailed in the first part of this paper shows that the evidence is extremely weak and unconvincing and does not warrant the interpretation placed upon it. My own considerable series of experiments, testing by many different ways the possibilities of natural infection, are in direct opposition to the widely accepted view that cats can suffer from a kind of diphtheria.

The evidence which has been adduced from the association of human diphtheria cases with cases of illness amongst cats whose throats are said to show diphtheria bacilli must be regarded as valueless in almost every case, for the following two reasons.

In all the instances that I have been able to find, with the exception of one case recorded by Barras and possibly Case 5 of my series, the diagnosis rests entirely upon the presence in the throat of the cats of bacilli morphologically like $B$. diphtheriae. Barras does not mention the steps he took to identify his bacillus and one cannot exclude the possibility of it being a pseudo-diphtheria bacillus. The results recorded above show that in normal cats no less than 66 per cent. showed bacilli which without isolation and study in pure culture I was unable to exclude as not true diphtheria bacilli. In my series of cats associated with cases of human diphtheria both Cases 1 and 4 would without doubt have been classed and accepted as proved cases of diphtheria in cats although a more complete investigation quite refuted this assumption.

The second reason is that the fact that the cat or cats were ill does not supply even prima facie evidence for suggesting that the human and cat conditions were related.

It is recognised by veterinary authorities that cats suffer from illness with symptoms which in some respects are analogous to those of human diphtheria but which are certainly not cases of diphtheria or due to $B$. diphtheriae. 
Gray (1896), for example, states that the so-called diphtheria in the cat is a contagious disease of the cat, characterised by the presence of diphtheritic membranes on the fauces, pharynx or larynx and due to some nicro-organism not yet determined. The disease has a mortality of quite 90 per cent.

Gofton (1913) says that the kidney condition described by Klein as pathognomic is an exceedingly common condition of the cat's kidney. He adds "affections presenting diphtheritic characters are met with in the cat and occur independently of human diphtheria, but the bacteriological investigation of these affections has up to the present always resulted in a failure to demonstrate the presence of the human bacillus." With a prevalent disease such as human diphtheria and a not very rare illness of cats with the above symptoms it is obvious that the two will be associated together in a certain proportion of cases. I investigated my first cases of supposed cat diphtheria (with negative results, not recorded here) just over 20 years ago and I have been on the look out for associated cases ever since and undoubtedly the association of the two conditions is infrequent. In fact we shall probably be correct in affirming that the two diseases are not associated together in any higher proportion than the relative frequency of the two conditions mathematically postulates. There are therefore no grounds for the assumption so commonly made that when the two conditions do happen to coincide we should assume a relationship of cause and effect between the two.

The interesting cases of illness in cats recorded by Webb (loc. cit.) are clearly examples of this infectious disease in cats and there is no justification for dragging in the human factor to account for them.

My experimental work affords no support to the suggestion that the cats may act as carriers by the bacilli living in their throat or nose. On the contrary it would appear that the mucous membranes of these animals are particularly inimical to these bacilli and that even under the most favourable circumstances they are unable to multiply or even survive for more than a trifling period. That the fur of these animals may be infected with these bacilli is of course quite a possibility and there is some experimental evidence in its favour (e.g. Remlinger, 1906) but in view of the low resistance powers of $B$. diphtheriae this is probably an unimportant factor in the spread of the disease.

Summing up the matter I am of opinion that the common and widely accepted view that cats can suffer from a naturally acquired disease caused by the diphtheria bacillus is entirely without foundation.

The reported cases of such an association are based upon insufficient examination and differentiation of the bacilli due to a failure to realise that a large proportion of healthy normal cats contain in their throats bacilli which closely resemble and are difficult to distinguish from the true $B$. diphtheriae. 


\section{REFERENCES.}

BARRAS, W. G. (1905). Lancel, ㅍ. 1869.

(1906). Public Health, xvIII. 709.

Dowson, W. (1895). Report of Medical Officer of Health, Bristol.

Gotton, A. (1913). In Hoare's System of Veterinary Med. I. 310.

GRAY, H. (1896). Journ. of Comp. Path. and Therap. IX. 46.

- (1913). In Hoare's System of Veterinary Med. r. 317.

GwYnn, E. (1893). Brit. Med. Journ. II. 1013.

KLeIN, E. (1888). Report of Medical Officer, Local Government Board, 435.

(1889). Ibid. 156.

(1890). Ibid. 229.

Low, BRUCE (1888). Ibid. 131.

Mapleton, H. B. (1913). Annual Report Newton Abbot Urban District Council, and personal communication.

Porter, A. E. (1908). Brit. Med. Journ. I. 622.

Primstley, J. (1915). The Medical Officer, June 5th, 253, and personal communication.

Remuinger, P. (1906). Revue Scientifique, Dec. 29th, 801. Summary. Brit. Med. Journ. 1907 , I. 552.

Renshaw, C. J. (1885). Brit. Med. Journ. I. 12.

SYMrS, J. O. (1896), Ibid. I. 1385.

TURner, G. (1886). Report of Medical Officer, Local Government Boasd, 307.

WEBB, F. (1914). Journ. Royal San. Inst. xxxv. 63, and personal communication.

WeLCH and Aввотт (1891). Johns Hopkins Hosp. Bull. II. 25.

Wrlliams, W. (1895). Brit. Med. Journ. ㅍ. 74. 